\title{
Germanica
}

\section{Allemands et Polonais dans Le Moulin à Lévine de Johannes Bobrowski}

Deutsche und Polen in Levins Mühle von G. Bobrowski

Poles and Germans in J. Bobrowski's novel: Levins Mühle

Jean Chabbert

\section{CpenEdition}

Journals

Édition électronique

URL : http://journals.openedition.org/germanica/2776

DOI : 10.4000/germanica.2776

ISSN : 2107-0784

Éditeur

Université de Lille

Édition imprimée

Date de publication : 31 décembre 1987

Pagination : 65-80

ISSN : 0984-2632

Référence électronique

Jean Chabbert, «Allemands et Polonais dans Le Moulin à Lévine de Johannes Bobrowski », Germanica

[En ligne], 2 | 1987, mis en ligne le 16 février 2015, consulté le 06 octobre 2020. URL : http://

journals.openedition.org/germanica/2776 ; DOI : https://doi.org/10.4000/germanica.2776

Ce document a été généré automatiquement le 6 octobre 2020.

(c) Tous droits réservés 


\title{
Allemands et Polonais dans Le Moulin à Lévine de Johannes Bobrowski
}

\author{
Deutsche und Polen in Levins Mühle von G. Bobrowski
}

Poles and Germans in J. Bobrowski's novel: Levins Mühle

\author{
Jean Chabbert
}

1 «Peut-être est-ce une erreur de raconter maintenant comment mon grand-père a provoqué l'inondation qui a emporté le moulin, mais peut-être aussi n'en est-ce pas une, même si cette histoire retombe sur la famille. »

2 C'est ainsi que Johannes Bobrowski commence son roman. Ce fait divers pourrait très bien se passer dans n'importe quel coin de campagne, entouré du secret qui empêche la découverte par des tiers d'une vérité que chacun connaît dans le groupe humain du village, et par là empêche la justice de châtier le coupable. Mais il s'impose à l'auteur comme thème d'une œuvre romanesque car il ressent le poids de cet héritage familial, une responsabilité diffuse - les péchés des pères ne retomberont-ils pas sur les enfants jusqu'à la troisième ou quatrième génération?

Cet héritage familial encombrant n'est en rien comparable aux conséquences d'une banale querelle de voisinage entre paysans. Il s'inscrit dans l'histoire d'une région qui connut au cours des siècles de durs affrontements entre nationalités, la plaine traversée par le cours inférieur de la Vistule. L'histoire de son grand-père n'est qu'une variante à l'échelle de la vie quotidienne d'un village du passé de son peuple dont il ne peut se débarrasser. Pour comprendre à quel point fut impérieuse la nécessité de cette confrontation, de cette expiation par le récit, il faut se souvenir de ce que Bobrowski déclarait dans une conférence prononcée devant l'académie protestante de la province de Berlin-Brandebourg le 21 décembre 1962 :

Le matériau que travaille la littérature, c'est le passé, le passé au sens le plus large du terme, donc aussi ses manifestations périmées; elle le fait avec le regard fixé sur

le présent et, pourquoi pas, sur l'avenir' ${ }^{1}$. 
Il faut aussi se souvenir de ce que fut sa vie dans le contexte qui suscita ce sentiment de culpabilité. Bobrowski fut emporté par la maladie le 2 septembre 1965 à l'âge de quarante-huit ans, alors que ses écrits - notamment Levins Mühle, publié en 1964 commençaient à connaître la renommée en RDA dont il était citoyen mais aussi en RFA. Il était né à Tilsit en 1917, près de la frontière lithuanienne, dans une famille descendant de paysans de Prusse-Orientale établis sur des terres conquises quelques siècles plus tôt par l'Ordre des Chevaliers Teutoniques en un lieu où vivaient des Polonais, des Lithuaniens, des Russes, des Allemands et parmi eux tous des juifs. L'étudiant d'histoire de l'art qui appartenait à la «Bekennende Kirche ", l'Église confessante qui regroupait l'opposition protestante au nazisme, fut incorporé dans la Werhmacht et connut la guerre en Russie. "Là, j'ai pu voir de mes yeux ", déclarait-il dans une interview de septembre 1964 diffusée sur le Deutschlandsender, « ce que l'histoire m'avait appris de la lutte de l'Ordre des Chevaliers Teutoniques contre les peuples de l'Est et ce que je savais de la politique de la Prusse dans les territoires de l'Est. C'est uniquement pour ce thème que j'ai commencé à écrire. Je l'ai considéré comme mon thème $»^{2}$. Une fois la paix revenue, il voulut témoigner par l'écriture, non sur ce que fut son vécu direct, mais sur les pratiques de son peuple dans le passé.

5 Le roman Levins Mühle, Le Moulin de Lévine, devait être une des composantes de cette confrontation avec le passé. Néanmoins, le lecteur ne doit s'attendre ni au récit de luttes sanglantes, ni à la description de scènes de destruction ou de pillage. C'est dans un village qu'il a situé l'action, dans une période de paix entre les nations, ses personnages incarnent la banalité d'un milieu rural considéré comme protégé contre les phénomènes de masse qui exacerbent les passions et l'agressivité individuelles, mais où peuvent émerger dans les représentations et pratiques quotidiennes ce qui nourrit ces phénomènes de masse. En quoi réside donc l'intérêt de ce récit dans la problématique qui nous occupe ici, « ethnicité et figures phobiques»?

6 La réponse à cette interrogation peut nous être fournie par l'analyse du milieu dans lequel vivaient ces villageois et des événements qui s'y passèrent en cette année 1874, par l'étude des clivages entre ethnies et cultures différentes et de leur exploitation par les protagonistes de l'action. Toutefois, Bobrowski considère que le conflit entre nationalités n'est qu'une composante d'un problème plus vaste, une justification commode d'autres clivages et contradictions, l'appartenance à une ethnie ou à une culture différente légitimant le rejet de ceux qui pour d'autres raisons moins avouables sont jugés indésirables par le groupe dominant. Quelle est donc la fonction de cette catégorisation si commode fondée sur l'appartenance à une ethnie? Les représentations qu'elle véhicule et les comportements qu'elle induit ne sont-ils que des prétextes? Quelle leçon l'auteur tire-t-il de cette chronique villageoise?

Quel est donc le milieu dans lequel Bobrowski a situé l'action de son roman? Une région située dans le pays de Culm, sur la rive droite de la Vistule, qui est devenue prussienne en 1772 lors du premier partage de la Pologne ; c'est une région agricole avec de nombreux villages disséminés dans la campagne, comme Neumühl au bord de la rivière Drewenz, le théâtre de l'action, ou Malken ; à proximité, il y a la petite ville de Briesen, tous les chemins mènent à Briesen, car c'est le chef-lieu administratif. La terre est fertile et produit des céréales, aussi le meunier est-il un personnage important dans ce milieu à côté des gros paysans propriétaires du sol dont les granges et greniers attestent la richesse - et surtout si le meunier est lui-même gros propriétaire, ce qui est 
le cas pour le personnage principal du roman, celui que Bobrowski appelle son grandpère, le vieux Johann.

Ces paysans étaient venus de Prusse avant même l'annexion. C'est ainsi qu'un généalogiste a trouvé trace de l'arrivée d'une famille Bobrowski en 1739 dans le village de Malken. En effet les nobles polonais avaient offert des terres à des paysans prussiens de confession luthérienne bien avant l'installation de l'administration prussienne et une pratique de germanisation active. Notre village de Neumühl est donc habité en majorité par des Allemands, mais Bobrowski prend soin de préciser tout de suite, pour éviter des malentendus : les paysans les plus gros étaient des Allemands, les Polonais du village étaient plus pauvres, pas aussi pauvres toutefois que ceux qui vivaient dans les villages de bois des alentours ${ }^{3}$. C'est peut-être à cette caractéristique qu'on pouvait distinguer les uns des autres car les noms de famille ne prouvaient rien : les Allemands s'appelaient Kaminski, Tomaschewski, Kossakowski, Ragolski, Wistubba ou Koschorreck, et les Polonais Lebrecht, Tethmeyer ou Germann ${ }^{4}$. Une autre caractéristique pour les distinguer: la religion, les Allemands étaient luthériens ou baptistes, méthodistes ou adventistes, les Polonais catholiques.

Mais Allemands et Polonais n'étaient pas les seules composantes de la population, il y avait aussi des juifs comme Lévine, des gitans comme le joueur de violon Habedank, des individus qu'on ne pouvait rattacher à aucune ethnie ou confession, le vagabond et chanteur Weiszmantel, le poète et joueur de flûte Johann Wladimir Geethe, le marginal Willuhn, qui fut naguère instituteur mais que son ivrognerie a perdu, sans oublier un cirque italien installé dans la forêt toute proche. Il semble que soit ainsi esquissée l'image du chaos racial que les nazis dénonceraient, ce chancre de l'Europe que constituait la Pologne. Ce village n'a du reste rien de spécifique: l'action, note Bobrowski, aurait aussi bien pu se passer en d'autres lieux, cent kilomètres plus à l'est, en d'autres temps. Pourtant, le cadre historique est largement évoqué ${ }^{5}$.

Lorsque l'action débute, nous sommes en 1874. Pourquoi l'auteur l'a-t-il située cette année-là ? Bismarck vient de réaliser l'unité allemande. Conscients de leur puissance en Europe, les Allemands se comportent à présent comme des maîtres sûrs d'eux-mêmes dans les territoires récemment annexés ou à forte population étrangère. Ils s'avancent avec fierté vers un avenir serein : il suffit de regarder le vieux Johann trônant sur sa calèche lorsqu'il se rend à Briesen.

Il a de la prestance maintenant, surtout le dimanche, lorsque la chaîne en or de sa montre orne son ventre, surtout lorsqu'il est saoul et que son foie se gonfle sous son gilet. Un homme et un Allemand, et mon grand-père !

C'est dans ce contexte et dans cette atmosphère qu'une politique est menée par le gouvernement du Reich pour résoudre le problème des minorités : conscient de sa force, celui-ci sait aussi que les minorités victimes de cette politique ne pourront plus trouver du soutien auprès d'autres puissances européennes tant le Reich dominateur contrôle l'équilibre entre celles-ci. De plus, la politique du Kulturkampf est l'occasion de réprimer plus durement les aspirations de la population polonaise qui ne cesse de se référer au soulèvement de 1863 dans l'autre partie de la Pologne sous domination russe. Lorsque les différentes tendances et sectes du protestantisme s'unissent à Malken, quelles qu'aient pu être leurs divergences antérieures, notamment sur la question du baptême, c'est pour contribuer à ce que le Reich « conduise jusqu'à son terme glorieux l'œuvre de notre Docteur Martin Luther ». Et comme le pasteur Glinski avait ajouté : 
« Mais, Messieurs, c'est dirigé contre l'ennemi, le Siège romain et c'est-à-dire... » le vieux Johann complète la déclaration inachevée : « contre les Polaques! \$ ${ }^{7}$.

$\mathrm{Au}$ nom du Kulturkampf se ravive l'animosité contre les Polonais car de toute façon catholique et polonais étaient synonymes. Très concrètement, le pouvoir prussien s'attaque au clergé polonais à qui il retire par la loi de 1872 tout contrôle sur l'école, à la langue polonaise par l'ordonnance de 1873 qui rend l'allemand langue obligatoire pour l'enseignement dans les écoles primaires avant qu'il devienne en 1876 la seule langue de l'administration. Reste ce que le Regierungspräsident, préfet de la région, qualifie de situation chaotique, mais une loi sur la colonisation est en préparation qui va y mettre bon ordre: "Ce sera alors fini, plus de Polaques dans les villages allemands $! »^{8}$. Tandis que les paysans polonais seront expropriés, leurs fils iront travailler dans les charbonnages de la Ruhr.

Les années 70, les Gründerjahre, constituent donc un tournant dans la politique à l'Est du nouveau Reich: tout ce qui est polonais doit être combattu, y compris par des initiatives individuelles propres à accélérer le processus de refoulement de l'influence polonaise, même en recourant à la violence et le cas échéant à des moyens délictueux dont on est persuadé qu'ils seront compris et couverts par l'administration prussienne, car, après tout, on est allemand et la victime fait partie de ce chaos ethnique qui peuple encore les campagnes.

C'est l'histoire d'une de ces initiatives individuelles que nous conte Bobrowski, un banal règlement de comptes entre paysans, mais qui prend ici une signification particulière.

Lorsque l'action commence, nous savons déjà qu'un moulin a été emporté par les eaux d'un petit affluent de la Drewenz. Et celui que le narrateur appelle son grand-père, « un homme et un Allemand », le vieux Johann, propriétaire d'une ferme et d'un moulin, a encore sur son terrain les restes d'un barrage qui avait retenu l'eau jusqu'à ce que cette masse soit libérée et emporte le moulin de Lévine situé en aval. Des pieux, des planches, des fascines de branchages jonchent encore le lit de la rivière. Furieux, il s'en prend à ses deux ouvriers polonais, il leur avait pourtant bien dit d'enlever tout ça! Au printemps 1874, il a en effet libéré lui-même cette masse d'eau pour détruire le moulin de son concurrent juif Lévine. Nous sommes à présent en été de la même année et il s'efforce de prouver qu'il n'y est pour rien. Mais cela devient de plus en plus difficile car Lévine a vu ce qui reste de ce barrage de fortune et des rumeurs circulent avec une insistance croissante.

16 Il faut donc qu'il agisse car Lévine a déposé plainte et l'affaire doit venir devant le tribunal de Briesen. Lévine obtiendra-t-il réparation? le vieux Johann sera-t-il condamné ? La menace se précise : une seule solution semble envisageable : faire retirer la plainte de Lévine. C'est alors que le vieux Johann mobilise toute la population allemande dans une association qu'il crée, l'« Union de Malken de 1874 ». Oubliées toutes les querelles et divergences qui l'opposaient lui, le doyen de la communauté baptiste de Neumühl, au pasteur luthérien de Malken dont il avait donné par dérision le nom à son jars! Il a besoin du pasteur pour obtenir le soutien du juge du tribunal de Briesen et du Landrat, le sous-préfet de l'endroit. N'y va-t-il pas en effet du combat qu'il faut mener au nom de la nation allemande contre ces juifs et ces Polaques qui menacent de submerger les Allemands?

17 Sur une intervention du pasteur, le procès est renvoyé une première fois, ce qui donne le temps au vieux Johann de tenter de neutraliser les témoins à charge possibles. En 
premier lieu Habedank le gitan qui rôde partout, épie tout et semble en savoir trop long. Il n'y a rien de plus facile dans le cas de cet individu en marge de la société. La vieille chaumière qu'il habite indûment a été saisie par les Domaines après la disparition de son ancien propriétaire, elle est mise en vente, le vieux Johann s'en porte acquéreur, fait expulser Habedank et met le feu, une nuit, à la bâtisse. Tout semble accuser le gitan : par vengeance, il serait devenu incendiaire. Son arrestation pour l'enquête rend impossible tout témoignage dans l'affaire du moulin. Quant aux autres témoins, il ne reste plus qu'à les discréditer : peut-on vraiment faire confiance à ces Polaques? la parole de bons Allemands, pieux et bien-pensants, n'est-elle pas plus digne de foi ? Et ce qui devait arriver arriva; le procès eut bien lieu, mais aucun des témoins n'avait vu ce qui s'était passé. Découragé, Lévine demande un nouvel ajournement mais, ruiné, ayant perdu tout espoir en la justice, il quitte le pays pour la Polonge-russe voisine - là où il est mieux à sa place, disent ces messieurs de Briesen. Il ne reste plus au juge qu'à clore le dossier : le plaignant a disparu.

Notre vieux Johann, « un homme et un Allemand», pourra-t-il jouir de sa victoire? Certes, Lévine et son amie Marie sont partis, mais ceux qui l'avaient soutenu sont restés, ouvriers agricoles, Polonais, gitans, gens du cirque, tous ceux qui sont bien décidés à réagir contre les agissements de ces bons Allemands qui, parce qu'ils ont du bien au soleil, se croient au-dessus des lois. Et ils vont faire en sorte que notre meunier ne puisse jouir de sa victoire. La fête des moissons est l'occasion rêvée : les deux camps se trouvent face à face; Habedank, relâché de sa prison car il était bien loin de Neumühl le soir de l'incendie de la chaumière, est là avec son violon, Weiszmantel avec ses chansons et le poète Johann Wladimir Geethe avec sa flûte. La fête peut commencer : du travail proprement fait, pas une table renversée, pas un verre cassé dans la salle de l'auberge de Rosinke, mais tous les bons Allemands, copieusement rossés au son du violon et de la flûte, volent par la porte et les fenêtres ouvertes. Johann Wladimir Geethe peut tirer la conclusion de cette nouvelle bataille de Valmy :

Ça, c'est nouveau! et nous, pauvres damnés de chiens, nous pourrons dire, sacrebleu, que nous y étions, tonnerre de Dieu !9.

19 La nouveauté, c'est que le vieux Johann va quitter Neumühl avec armes et bagages pour se retirer dans un trois-pièces à Briesen, à la ville où il pourra assouvir son ressentiment en devenant, lecteur assidu de la Gartenlaube, un propagandiste acharné de l'antisémitisme au service du nationalisme. Et il écrira même à Otto Glagau, rédacteur de cette même Gartenlaube, pour lui faire part de ses expériences et lui proposer des solutions au problème juif en Pologne. Mais un autre artiste surgira sur son chemin, le peintre Philippi ; il avait bondi devant lui sur un trottoir. «Laissez-moi donc tranquille», s'était écrié le vieux, "mais non!» avait répliqué l'autre en lui claquant les mains sous le nez, et ce «non! » est la dernière phrase du roman.

Voilà bien un heureux dénouement ! l'auteur des forfaits est puni, tous ceux qui étaient discriminés, bafoués, se sont vengés. Mais est-ce bien là l'essentiel dans le roman? Bobrowski y aborde deux thèmes importants : celui du rôle de l'artiste dans la lutte contre l'injustice et l'inhumanité, sa force et ses limites, par le truchement, dans ce milieu campagnard, de ces musiciens, chansonniers et poètes de village - mais ce n'est pas notre propos ici -, et celui de l'émergence, de la propagation et de l'exploitation de représentations négatives de l'autre lorsque par son origine nationale ou sa culture il est considéré comme ne faisant pas partie du groupe. 

sont pas nées dans l'esprit de nos paysans allemands bien-pensants et pieux : elles ont une histoire qui imprègne la conscience collective. Frédéric II n'hésitait pas dans son Testament politique à considérer que la Pologne ne faisait pas partie des puissances européennes; il déniait le droit à la nation polonaise de se constituer en État et il comparait ces territoires au Canada de l'époque et les Polonais aux Iroquois. Nombre d'intellectuels de l'époque, même parmi les philosophes des Lumières, se réjouissaient, après le partage de la Pologne de 1772, que l'esprit éclairé de l'administration autrichienne ou prussienne ait mis bon ordre à la barbarie de ces peuplades. Les seules critiques formulées contre ce partage ne l'étaient pas au nom du droit à l'existence d'une nation mais par crainte d'une rupture de l'équilibre des forces en Europe. L'interprétation de l'histoire humaine comme résultat de l'affrontement entre races de valeur inégale, telle qu'elle se développa ensuite dans la deuxième moitié du XIXe siècle, ne fit qu'apporter de nouveaux contenus à ces représentations. Face aux Germains qui seraient la race pure et créatrice de culture et de valeurs, les Slaves ne seraient que des dégénérés, une situation aggravée par la prolifération des juifs sur ces terres dont ils feraient leur base de départ pour la mainmise sur l'Europe. C'est donc en toute bonne conscience que nos braves paysans allemands se considèrent comme les défenseurs du Reich et de la civilisation.

Ils ne doutent pas un seul instant d'ailleurs dans la vie de tous les jours de leur supériorité. Ces Polaques sont des paresseux et des propres-à-rien. Dès que le vieux Johann a le dos tourné, ses deux ouvriers polonais au moulin cessent de travailler, hument le parfum des fleurs ou prisent leur tabac. Il peut bien donner des ordres, ils n'en font rien: il faut surgir à l'improviste derrière une haie pour les surprendre et après chaque absence il faut compter les sacs de farine pour savoir s'ils ont travaillé. Et que font-ils lorsqu'on les réprimande? ils continuent à priser leur tabac, assis tranquillement au soleil devant le moulin. Heureusement, dès qu'ils deviennent insupportables, on les renvoie, qu'ils aillent donc là-bas, en Pologne russe! Que font les autres Polaques? ils sont ouvriers agricoles, valets de ferme, tâcherons. Mais personne ne parle de leur travail, on sait à quoi s'en tenir, il n'y a rien à attendre d'eux. Pourtant, lors de l'embauche annuelle des valets de ferme à la Saint-Michel, n'ont-ils pas l'impudence de poser des revendications? Ce n'était pas ainsi autrefois, le salaire était d'un taler, et il n'y avait pas de discussion. " C'était le paradis, dit mon grand-père, mais ça tient à la crainte de Dieu qui diminue! $»^{10}$.

Que pourrait-on du reste attendre de ces individus en qui on ne peut avoir aucune confiance? tous les bons Allemands savent qu'ils ne disent jamais la vérité, qu'ils sont fourbes, hypocrites, sournois. A priori la justice et la police considèrent le témoignage d'un Polonais comme suspect. Lorsque Lé vine demande une audition de Korrinth et Nieswandt sur les agissements du vieux Johann, le juge Nebenzahl lui répond: «des Polonais? et vous persistez dans votre demande, Monsieur Lévine? $»^{11}$. Cette question empreinte de mépris caractérise la prévention du juge à l'égard de ces individus dont le témoignage ne peut être que mensonger. De même que le témoignage de Weiszmantel sur la présence de Habedank le gitan au moment de l'incendie de la chaumière dans une auberge de Strasburg. Il n'a pas de domicile fixe et il prétend témoigner? demande réjetée. Pour l'administration il y a bien des individus qui ne peuvent être assimilés aux citoyens à part entière. 
Comment pourrait-on respecter ces gens-là puisqu'on leur refuse même le droit à une identité culturelle? Nos braves Allemands se moquent à qui mieux mieux de la langue qu'ils parlent - bien que leur propre langue soit émaillée d'expressions polonaises! Une langue qui n'a pas de nom du reste puisqu'on n'éprouve pas le besoin d'identifier ce qui n'a pas de consonance allemande. Quel jargon parle Antonja, la montreuse de coqs savants du cirque ? C'est de l'italien ou du polonais, on ne sait pas exactement, à quoi bon chercher à savoir si c'est de l'italien ou du polonais ${ }^{12}$ !

En fait, nos bons Allemands, à l'instar du vieux Johann, se demandent bien ce que font ces Polonais dans ce pays en cette année 1874. Rien ne l'exaspère plus que le rappel des coutumes et dictons polonais. "Toujours ces foutues histoires de Polonais! autrefois en Pologne! autrefois en Pologne, mais qu'est-ce que ça signifie "autrefois en Pologne" ? Mais partez-y donc dans cet autrefois, dans cette Pologne! $»^{13}$. Plus direct est le chef d'escadron von Lojewski, le vieil ivrogne. Ne s'est-il pas indigné dans l'auberge Deutsches Haus qu'on puisse jeter par la fenêtre de la bière allemande en la servant aux polaques? n'a-t-il pas déclaré qu'il n'y avait plus d'honneur allemand si ces Polaques avaient de la bière au même prix que les honnêtes gens ? n'a-t-il pas clamé haut et fort qu'il ne pouvait pas être vrai qu'ils puissent trâner partout ici, ces Polaques, comme du sable dans la mer ${ }^{14}$ ?

Mais c'est lors de la fête des moissons que le processus d'exclusion va fonctionner à plein, entraînant la réaction des autres. Les Polonais, cette canaille, cette racaille, sont déjà assis dans la salle de l'aubergiste Rosinke lorsque le vieux Johann, accompagné de son clan, y pénètre. Son regard fier fait le tour de la salle, sa décision est prise : «Je ne vais pas m'asseoir avec des Polonais!» s'exclame-t-il. Et comme son beau-frère veut payer une tournée aux musiciens, c'en est trop! Il hurle dans la salle à l'aubergiste : «Mais pas pour les Polaques! » Par solidarité avec les Polonais, le poète Geethe refuse le verre de schnaps qui lui est offert. C'est le signal de la bagarre. «Foutons dehors cette racaille! nous verrons bien qui a son mot à dire ici!" s'écrie le bon Allemand Tomaschewski. Mais nous savons que c'est la racaille qui eut le dernier mot. Victoire trompeuse cependant car ce qui s'est passé à Briesen au tribunal était d'une autre importance. Le vieux Johann avait obtenu finalement que le procès n'eût pas lieu ${ }^{15}$.

Car le juif Lévine était dans une position encore plus inconfortable que ces Polaques catholiques. N'est-il pas celui qui est venu de nulle part, cet errant faisant brusquement son apparition ici, avant de disparaître à nouveau? Personne ne s'étonne d'ailleurs qu'il ait ainsi disparu, peut-être est-il dans la Pologne russe ? En tout cas, il est mieux à sa place là-bas, de l'autre côté de la frontière. N'est-il pas un de ceux qui portent sur leur front le signe de Caïn, la marque indélébile des meurtriers du Christ et qui rôdent par le monde à présent? Même parmi les Polaques il est suspect. N'est-il pas celui qui fait coûte que coûte des affaires et qui prend leur travail aux honnêtes gens? N'est-il pas un de ceux qui arrivent «le cul nu et qui font des affaires? ${ }^{16}$. Ce Lévine avait appris quelques bribes du métier de meunier, avait bricolé un moulin, quatre pieux, quelques poutres et des planches là-dessus, une roue; cela suffit pour faire de bonnes affaires et retirer sa clientèle au vieux Johann. Car il avait compris que les paysans des environs avaient besoin d'argent frais tout de suite : il leur avait acheté le grain, les avait payés immédiatement et avait revendu lui-même la farine au lieu de laisser cette vente à la charge des paysans comme c'était la pratique courante dans les autres moulins. 
28 Ainsi émergent dans le discours des différents personnages du roman trois des stéréotypes qui s'attachent à l'image du juif dans l'antisémitisme vulgaire : le type sans patrie, l'assassin du Christ et l'affairiste. Mais là ne réside pas seulement l'intérêt du texte : il réside plutôt dans le fait que ces stéréotypes sont propagés sans doute par les bons Allemands mais aussi par la population polonaise catholique, par ceux-là mêmes qui sont déjà discriminés pour leur origine ethnique. Pourraient-ils du reste les mettre en doute ? Ils ont été répétés tant de fois qu'ils sont des vérités évidentes et chacun est sûr d'en avoir rencontré la preuve dans sa vie. En outre, il est si réconfortant de pouvoir, étant méprisé soi-même, mépriser quelqu'un d'autre! Seuls quelques-uns parmi les méprisés et discriminés sont capables de percevoir le danger d'une telle attitude, Weiszmantel et Habedank, les artistes à l'échelle du village, car elle aboutit à la division de tous ceux qui sont opprimés par les bons Allemands. Prendre la défense de Lévine, c'est donc affaiblir la position de ceux-ci, c'est aussi lutter contre l'injustice sous toutes ses formes qui frappent les Polonais.

29 Car le cas de Lévine est exemplaire: il n'a rien à attendre de la justice et de l'administration prussiennes dans son affaire. D'ailleurs, la femme de Rosinke, l'aubergiste, s'indigne même de ce qu'il ait pu déposer plainte pour la destruction de son moulin. Pour qui se prend-il donc ce juif pour déposer plainte contre le vieux Johann, le doyen de la communauté baptiste de Neumühl? Personne n'a rien vu, explique-t-elle au gendarme Krolikowski, car elle est persuadée que le mécanisme d'exclusion fonctionnera à plein et que personne ne soutiendra le juif. Mais ce ne fut pas le cas. Habedank - mais ce n'est qu'un gitan que le vieux Johann voit avec colère toujours rôder dans la campagne - voulut dénoncer le forfait et dire ce qu'il avait remarqué. Réaction du vieux Johann :

S'il veut vivre en paix, qu'est-ce qu'il cherche celui-là? C'est sans doute ce qu'il veut, vivre en paix. Et voilà qu'il se mêle de cette histoire avec le juif. Qui lui a demandé quelque chose ? $^{17}$

C'est la récompense que l'on a lorsqu'on laisse ces types-là s'installer quelque part. Et la tante Huse aussi - on l'appelait ainsi dans le village, mais on ne savait plus très bien de qui elle était la tante - voulut dénoncer le forfait. Le greffier du tribunal ne peut s'empêcher de s'interroger : mais qu'est-ce que cette femme peut bien avoir avec ce juif ? de quoi se mêle-t-elle ? Et comme elle persista à vouloir dire ce qu'elle savait, elle écopa une amende pour injure au tribunal.

31 Là est bien le problème. Toute l'administration, tous nos Allemands bien-pensants et pieux sont convaincus que tous les moyens sont bons, même lorsqu'ils portent atteinte aux droits des individus et à la vérité, pour s'imposer du moment qu'il s'agit de défendre l'identité et les intérêts allemands. Les conquérants qu'ils étaient dans ces territoires jouent le rôle de victimes qui seraient menacées par la poussée de ce conglomérat de peuples et de races qui mettraient en danger la germanité. Mais est-ce bien là la fonction de ces stéréotypes sur les autres et de ces pratiques?

Bobrowski n'y croit pas. Ces stéréotypes et comportements masquent en fait non des contradictions nationales mais des contradictions sociales. L'appartenance à un groupe ethnique ou culturel correspond à un statut social et à une position économique. Quant aux valeurs nationales et aux clichés dévalorisants pour les autres ethnies ou groupes culturels, ils justifient la position de chacun dans le contexte économique et social; la défense de ces valeurs n'est que le déguisement flatteur de la lutte pour le maintien d'intérêts économiques égoïstes, un prétexte pour parer cette lutte d'idéal. 

se cumule selon la définition que Bobrowski donne du personnage lorsque Weiszmantel le retrouve après une longue absence et constate un changement : conserver ce que l'on a, vouloir avoir plus et vouloir être meilleur que les autres ${ }^{18}$. Il est en effet celui qui à Neumühl possède le plus d'argent, il a un compte à la caisse d'épargne de KowalwoSchönsee sur lequel il a placé toute la dot de sa femme, 7000 talers, il possède des champs, un moulin. Il est celui qui n'a aucun sens pour la beauté de la nature mais qui estime tout ce qu'il voit, champs, arbres, plantes, en fonction de la valeur marchande et du rapport possible. Il est celui qui donne le ton à Neumühl et qui considère qu'il est au-dessus des lois. Aux gendarmes venus l'interroger, « instances subalternes », donc sans importance, il refuse toute déclaration, leur fait servir du schnaps par sa femme, "deux par personne ", et quitte la pièce. Que ces "serpents et pigeons en uniforme " aillent voir d'autres habitants de Neumühl! Il est en effet au-dessus de tous, en sa qualité de doyen de la communauté baptiste de Neumühl ${ }^{19}$.

ous ceux qui l'entourent, Tomaschewski et Kossakowski en tête, ont aussi du bien au soleil. Ceux qui vont se rallier à lui comme le Polonais Germann ou le Polonais Tethmeyer, le menuisier et fabricant de cercueils, ont aussi du bien ou veulent vivre en paix pour leurs affaires. Aussi n'a-t-il pas accepté que Lévine avec son nouveau moulin et ses pratiques commerciales particulières vienne le concurrencer dans ses affaires. Tout est parti de là : pour préserver son profit, il a détruit le nouveau moulin afin que, comme le dit si bien la tante Huse, « la bénédiction du Seigneur descende sur lui avec plus de magnificence! $»^{20}$. Il a ensuite réactivé par ses discours et son comportement toutes les réactions de discrimination ou de rejet à l'égard des autres, Polaques, gitans, juifs, pour pouvoir apparaître comme la victime de leurs machinations, comme le défenseur de la noble cause de la nation allemande menacée par tous ces ennemis. Ce qui était en fait en question, c'était son monopole de meunier à Neumühl.

Il n'était pas ainsi autrefois. Weiszmantel, qui l'avait connu dans le passé, ne le reconnut pas lorsqu'il le revit après une longue absence, tant il avait changé. L'appétit du profit et la volonté de puissance l'avaient transformé. Un appétit de profit que plus rien n'arrête à présent et qui, pour s'affirmer, a recours à la violation du droit et à la perversion des missions de l'État. S'avise-t-on de s'insurger là-contre ? il suffit alors de discréditer celui qui conteste ces méthodes et le meilleur moyen est d'exploiter les contradictions entre groupes ethniques ou culturels différents pour rassembler tous ceux qui veulent préserver l'ordre établi présenté comme base de l'identité nationale.

C'est là la leçon que Bobrowski veut tirer de cette histoire qui selon lui est représentative de l'histoire des peuples, de l'évolution des pratiques dans l'exercice du pouvoir. Poleske, l'une de ces apparitions qu'il arrive au vieux Johann de voir, surtout lorsqu'il a trop bu, est présenté comme son ancêtre. C'était un brigand de grand chemin qui rançonnait les marchands sur les routes de Pologne au début du $\mathrm{XvI}^{\mathrm{e}}$ siècle et utilisait la force pure pour satisfaire son appétit du gain. Le meunier de Neumühl vit, lui, à une époque qui sait pervertir toutes les valeurs et toutes les institutions. Il exploite l'idéal national pour défendre ses propres intérêts, quitte à se présenter comme la victime d'une machination alors qu'il est en fait considéré à juste titre par beaucoup comme un criminel et qu'une application sans faille de la loi châtierait comme tel. S'il parvient à ses fins, c'est parce que les représentations négatives des autres, les stéréotypes qui s'attachent à ceux qui sont d'une autre ethnie ou d'une autre 
culture, sont suffisamment puissants à l'état latent pour susciter des comportements qui masquent et travestissent la réalité, si banale soit-elle.

37 Seuls quelques individus parviennent à arracher ces masques et à dénoncer ces manœuvres, c'est-à-dire à prendre conscience des rapports sociaux réels. Mais ils risquent fort - quel que soit le groupe auquel ils appartiennent, Polonais ou Allemands, protestants ou catholiques - d'être de nouveaux exclus ou en tout cas dans une situation bien inconfortable, ce qui prouve bien à quel point ces représentations négatives n'ont aucune valeur absolue mais une fonction purement conjoncturelle.

\section{NOTES}

1. - Johannes Bobrowski, Selbstzeugnisse und Beitrage über sein Werk, Union Verlag (textes réunis par Gerhard Wolf, Berlin, 1966), p. 27.

2. - Ibid., p. 51.

3. - Johannes Bobrowski, Levins Mühle, 34. Sätze über meinen Grossvater, Berlin, Union Verlag (6 éd., 1969), p. 6 (cité : LM).

4. - LM, cf. p. 6, 15.

5. $-L M$, cf. p. 295.

6. $-L M$, cf. p. 41.

7. - LM, cf. p. 59.

8. $-L M$, cf. p. 263.

9. - LM, cf. p. 258. Il s'agit évidemment de la parodie de la célèbre phrase prononcée par Goethe lors de la bataille de Valmy.

10. - LM, cf. p. 246.

11. - LM, cf. p. 216.

12. $-L M$, cf. p. 113.

13. - LM, cf. p. 66 .

14. - LM, cf. p. 171.

15. $-L M$, cf. p. 251-255.

16. - LM, cf. p. 176.

17. - LM, cf. p. 126.

18. - LM, cf. p. 92.

19. - LM, cf. p. 265.

20. - LM, cf. p. 134. 


\section{RÉSUMÉS}

Dans ce roman dont l'action se situe en 1874 dans les territoires de la Pologne partagée où se pratique une politique de germanisation active, Bobrowski nous montre, sur la base de faits historiques, comment la coexistence de populations d'ethnie et de culture différentes peut aboutir au mépris du droit et à la perversion de la mission de l'État. Stéréotypes dévalorisants et pratiques discriminatoires caractérisent les rapports entre Allemands, Polonais et juifs. Mais l'auteur relativise la justification par l'ethnie ou la culture de ces stéréotypes ou pratiques : il s'agit en fait du travestissement idéalisé de conflits économiques et sociaux.

In diesem Roman, dessen Handlung 1874 in den Territorien des geteilten Polen spielt, wo eine aktive Germanisierungspolitik durchgeführt wird, zeigt uns Bobrowski aufgrund historischer Ereignisse, wie die Koexistenz von Bevölkerungsgruppen mit unterschiedlichen ethnischen und kulturellen Eigenarten zur Verachtung bzw. zum Missbrauch des Rechts und zur Pervertierung der Aufgaben des Staats führen kann. Die Beziehungen zwischen Deutschen, Polen und Juden kennzeichnen sich durch herabsetzende Stereotype und diskriminierende Praktiken. Jedoch relativiert der Autor die Rechtfertigung dieser Stereotype und Praktiken durch ethnische oder kulturelle Argumente: es handelt sich in seiner Perspektive eigendich um eine ideologische Verklärung ökonomischer und sozialer Gegensätze.

The events narrated in this novel took place in 1874 in partitioned Poland, at a time of vigorous attempts at germanization. Bobrowski shows the reader how, on the basis of historical facts, the coexistence of ethnically and culturally différent populations can lead to a perversion of the role of the state and to the negation of individual rights. Derogatory stereotypes and discriminating behaviour being the typical of the relationships between Germans, Poles, and Jews. However, the author puts forward the idea that such behaviour is not ethnically or culturally grounded, but is a réfection of social and economic conflicts.

\section{AUTEUR}

JEAN CHABBERT

Université de Lille III 